\title{
L'efficacite D'un Programme Therapeutique Cognitivo-Comportemental Dans La Diminution Du Burn-Out Chez Les Infirmiers De L'hopital De Sfax (Etude Descriptive-Experimentale)
}

\author{
Dr. Galaa Telelaz \\ Psychologue clinicienne principale \\ Université de Tunis El Manar, Tunisie
}

Doi: 10.19044/esj.2018.v14n15p139 URL:http://dx.doi.org/10.19044/esj.2018.v14n15p139

\begin{abstract}
Burn-out result from exposure to chronic work stress, of which the caregivers and nurses are most exposed to this risk. That is why we conducted a descriptive-experimental study which is based on the quantitative approach which aims to describe the prevalence of burnout among our target population (the nurses of both public health institutions of the hospital of Sfax) and the indicators (self-esteem, coping strategy, locus of control, motivation, satisfaction, sense of self-efficacy, life orientation by a sense of coherence) that influence this syndrome and also to evaluate the effectiveness of a therapeutic program proposed and applied in this research on the reduction of the frequency of burnout. The hypotheses stipulate that there is a significant difference between the pre-test data and data collected at post-test among nurses in the experimental group. The results of our descriptive study show that $37.4 \%$ of participants suffer from high emotional exhaustion, 59.4\% suffer from high depersonalization and $48.5 \%$ suffers from a sense of lack of fulfillment in their work. $31 \%$ have a degree from low to very low self-esteem, (72.5\%) of participants with low levels of self-efficacy feeling an average motivation (being $50.3 \%$ ) to low (being $44.8 \%$ ) with $68.1 \%$ of a low amotivation, (44.8\%) with a low extrinsic motivation, (47.3\%) have low intrinsic motivation, $(70 \%)$ are neither satisfied / nor dissatisfied, $32 \%$ have a low sense of coherence, $52.9 \%$ use coping strategies focused on emotion and (56.4\%) seeking social support while $(75.3 \%)$ use weakly coping strategies focused on problem solving, a majority of them uses an external attribution (44\%) due to colleagues (42\%) due to the superior, and also (42\%) due to chance. The results of the experimental study responded generally to the research hypotheses given that they have shown significant differences between the two groups of nurses (experimental -control) post-test pre-test,
\end{abstract}


which affirms the effectiveness of treatment program in reducing burnout and improving indicators.

Keywords: Burnout, psychotherapy, cognitive behavioraltherapy, selfesteem, coping strategy, locus of control, motivation, satisfaction, sense of self-efficacy, life orientation, sense of coherence

\section{Résumé}

Le burn-out ou le syndrome d'épuisement professionnel est consécutif à l'exposition à un stress professionnel chronique et dont les personnels soignants sont les plus exposés à ce risque. L'étude de ce risque nous a amené à réaliser une étude descriptive-expérimentale qui se base sur l'approche quantitative dont le but est de décrire la prévalence du syndrome d'épuisement professionnel chez notre population cible (les infirmiers des deux établissements publiques sanitaires de l'hôpital de Sfax) ainsi que les indicateurs (estime de soi, stratégie de coping, centre de contrôle, motivation, satisfaction, sentiment d'auto-efficacité, l'orientation de vie par le sens de la cohérence) qui influent sur ce syndrome et aussi d'évaluer l'efficacité d'un programme thérapeutique proposé et appliqué dans cette recherche sur la diminution de la fréquence du burn-out. Les résultats de notre étude descriptive montraient que $37.4 \%$ des participants souffraient d'épuisement émotionnel élevé, $59.4 \%$ de dépersonnalisation élevée et $48.5 \%$ d'un sentiment de manque d'accomplissement dans leur travail. Près du tiers ( $31 \%)$ avaient un degré faible à très faible d'estime de soi, $(72.5 \%)$ des participants avaient un niveau faible de sentiment d'auto-efficacité une motivation moyenne (soit 50.3\%) à faible (soit 44.8\%) avec $68.1 \%$ d'une faible motivation, (44.8\%) d'une faible motivation extrinsèque, (47.3\%) avaient une motivation intrinsèque faible, $(70 \%)$ étaient ni satisfaits/ ni insatisfaits, $32 \%$ avaient un niveau faible de sens de la cohérence, $52.9 \%$ utilisaient des stratégies de coping centrées sur l'émotion et $(56.4 \%)$ cherchaient le soutien social alors que $(75.3 \%)$ utilisaient faiblement les stratégies de coping centrées sur la résolution de problèmes, une majorité d'entre eux utilisait une attribution externe (44\%) due aux collègues $(42 \%)$ due au supérieur et aussi $(42 \%)$ due à la chance. Les résultats de l'étude expérimentale ont répondu en général aux hypothèses de la recherche vu qu'ils ont mis en évidence des différences significatives entre les infirmiers des deux groupes (expérimental témoin) au pré-test et post-test, ce qui confirme l'efficacité du programme thérapeutique dans la diminution du burn-out et l'amélioration des indicateurs.

Mots Clés :Burnout, psychothérapie, thérapie cognitivo-comportementale, estime de soi, stratégie de coping, centre de contrôle, motivation, satisfaction, sentiment d'auto-efficacité, l'orientation de vie, le sens de la cohérence 


\section{Introduction}

Le travail occupe une place centrale dans le fonctionnement psychique d'un individu et dans la construction de son identité. (Estryn-Behar, 1997). Mais trop souvent il prend aussi une tournure détestable, temporairement ou durablement; des exigences physiques, des contraintes psychologiques, des tâches mal définies, et des conflits avec les collègues et les supérieurs. (Truchot ,2004)

Pour Loriol, (2001) et Piette, (2012), Le travail infirmier est perçu comme épuisant, fatiguant et angoissant vu la diversité des actes à accomplir par le soignant et la multitude des agents stressants auxquels il est confronté. Dans ce sens Phaneuf (2002) ajoute que malgré sa situation psychologique, l'infirmier se trouve devant un malade qui a besoin de se sentir accompagné et pris en charge, ce qui indique que le contexte de travail en soins infirmiers peut susciter des grandes difficultés et un stress (chronique) professionnel.

Le burn-out ou syndrome d'épuisement professionnel résulte en général d'une souffrance et d'énormes problèmes dans le milieu de travail comme la surcharge de travail, des relations difficiles ou des conflits personnels, l'absence de solidarité et de soutien social, de manque d'autonomie, des responsabilités mal définies et de l'insuffisance de la reconnaissance de leur travail. On relève que le personnel médical est plus exposé à faire l'expérience d'un stress émotionnel important causé par leur interaction constante avec des personnes malades ou ayant des problèmes, car ces situations sont très chargées en émotions. Les infirmières qui ont la sensation de donner plus à leur patients que ce qu'elles reçoivent en retour en termes de gratitude et d'appréciation rapportent des niveaux d'épuisement émotionnel, de dépersonnalisation et de diminution de l'accomplissement personnel très supérieurs à ceux des infirmières qui ne se sentent pas touchées par ce problème. (Van Yperen, Buunk, Schaufeli, 1992).

\section{Problématique}

Maslach \& Jackson (1986) affirment que Le burnout touche particulièrement les professionnels de la santé et dans les professions engagées dans une relation d'aide en raison, notamment, de leur implication intense et de longue durée auprès de personnes dans le besoin, réclamant des services.

Alors que Loriol (2001) a parlé de l'activité des infirmier(e)s en raison des responsabilités, du contact direct avec la souffrance, la mort et la saleté et d'une pratique professionnelle qui s'appuie sur des compétences difficiles à définir, l'activité infirmière est généralement perçue comme stressante et fatigante. La fatigue, même dans sa dimension physique, est souvent associée à la relation au malade. Ainsi, le mal de dos est autant lié au sentiment de ne pas pouvoir entretenir de bons rapports avec les patients qu'au port effectif de charges lourdes. 
Pasquier- Garnier (2012) explique que la personne qui souffre de burnout a l'impression d'être « vidée », d'avoir « brûlé ses réserves » : il lui reste juste assez d'énergie pour tenter de mener à bien son travail, au risque de sacrifier sa vie extra-professionnelle. Cet état s'accompagne d'une forte dépersonnalisation. L'infirmier(e) s'isole de son entourage, se sent inutile et impuissant, il se dévalorise mentalement, il a l'impression de ne plus satisfaire aux attentes professionnelles. Tous ces symptômes s'aggravent insidieusement et graduellement au fil des mois: un cercle vicieux se met en place, qui entraîne une baisse de l'estime de soi, un manque d'assurance et un fort pessimisme. Ce qui engendre une détérioration de l'accomplissement personnel au travail.

De plus, Rodary et Coll (1993) se sont penchés sur la souffrance des infirmiers à l'hôpital en menant une étude épidémiologique dans un centre anti cancéreux et un hôpital général en France. Ils ont relevé que sur un échantillon de 520 infirmier(e)s, il y a $25 \%$ d'entre eux qui souffrent d'un niveau élevé d'épuisement professionnel où les symptômes observés étaient le manque de soutien, un sentiment de peur du malade et le manque d'organisation de leur travail... (Estryn-Behar, 1997).

Un groupe d'expert de l'institut national de la santé et de la recherche médicale (2011) déclare que les prévalences d'épuisement professionnel varient beaucoup selon les études, de quelques pourcents à quelques dizaines de pourcents de $5 \%$ à $20 \%$ de la population d'étude.

Les données statistiques de l'Association Canadienne de Santé Mentale ont révélé que 50\% des personnes interrogées en 2001 indiquent que leur milieu de travail est une source majeure de stress contre seulement $39 \%$ en 1997 (Poirier \& Lafrenière, 2003, p 14.).

Devant ce phénomène d'épuisement professionnel, il est important de chercher des stratégies pour améliorer l'état psychologique des infirmiers et d'étudier profondément les facteurs en cause avec une évaluation du degré d'épuisement professionnel, et de bien décrire les composantes du burn-out (niveau organisationnel et individuel...). Il est important de procéder à une identification des éléments pour forcer les progrès en termes d'études longitudinales qui permet de mettre en place des programmes d'interventions et d'actions destinées à évaluer et traiter ce syndrome et permettre de diminuer son impact. La présente étude vise à mettre en évidence les liens pouvant exister entre le syndrome d'épuisement professionnel et d'autres variables psychologiques comme la satisfaction et la motivation, le sentiment de l'autoefficacité, le sens de cohérence, le coping, le centre de contrôle.

L'objectif de la TCC est d'aider les infirmier(e)s qui souffrent de burnout à retrouver leur autonomie et construire des relations interpersonnelles sans difficulté, à s'affirmer devant les situations stressantes par une habituation progressive, à travailler leur acceptation de soi par une évaluation 
positive de leur propre image, en repensant à leurs réelles qualités et potentialités, à lutter contre les conflits internes et les croyances négatives, à chercher des moyens alternatifs qui permettent d'identifier et de corriger les pensées automatiques.

Cette présente étude permet de s'intéresser à la problématique du burnout au niveau de l'hôpital de Sfax vu l'importance de ce problème et ses répercussions sur la productivité professionnelle et l'état psychologique des infirmiers. Il s'agit aussi de dynamiser la perception des infirmier(e)s sur ce sujet et les effets qu'il peut engendrer sur le rendement et la performance au travail.

Le but de cette étude est d'évaluer l'efficacité du programme thérapeutique cognitivo-comportemental dans la diminution de cette souffrance, de ce syndrome du burn-out chez les infirmiers des centres hospitalo-universitaires de Sfax par la comparaison des deux groupes (expérimental- témoin) concernant les données liées au burn-out: l'estime de soi, la satisfaction, la motivation, l'auto-efficacité, le sens de la cohérence, les stratégies de faire face (le coping), le centre de contrôle.

\section{Méthodologie}

\section{Devis de la recherche}

Cette étude transversale utilise le devis descriptif expérimental où on trouve un design de plan pré-test/post-test à deux groupes dans lequel on a appliqué à un groupe d'infirmiers souffrant de burn out un programme thérapeutique cognitivo-comportemental et, à la fin du programme, on a comparé les résultats obtenus avec les résultats del'autre groupe témoin « qui n'a pas participé à ce même programme ».Ce design nous a permis d'évaluer les effets du programme thérapeutique cognitivo-comportemental sur la diminution de cette souffrance, de ce syndrome du burn-out chez 30 infirmiers des centres hospitalo-universitaires de Sfax .

Pour répondre à notre question de recherche et réaliser les buts de l'étude, nous avons adopté un essai contrôlé randomisé de plan pré-test / post-test à deux groupes (témoin et expérimental), comme nous le montre la figure suivante: 


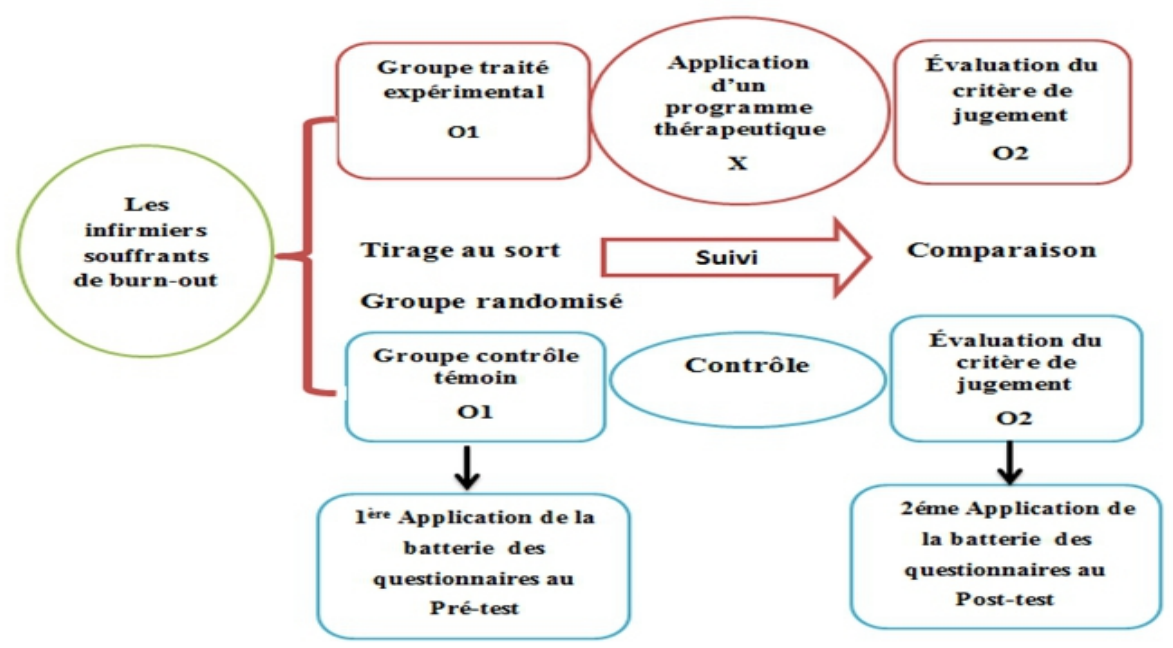

Figure 6 : Schématisation du devis de notre recherche

Nous avons utilisé une approche de recherche quantitative qui repose sur le recueil de données numériques de la batterie des questionnaires dans le pré-test et le post test. Le programme thérapeutique cognitivo-comportemental appliqué afin de comprendre les résultats de l'étude expérimentale, bien que les données des séances soit qualitatives, leur analyse garde une perspective quantitative et les résultats seront souvent exprimés en nombre selon les changements effectués sur les réponses des participants aux questionnaires, au pré-test et au post-test, afin de vérifier si les groupes sont différents avant le commencement et l'effet de la manipulation. (Murtagh, Thomson, May, Rapley, Heaven, Graham, Kaner, StobbartetEccles, 2007).

\section{Population et échantillon}

La population de l'étude était composée des infirmiers de l'hôpital de Sfax, la méthode d'échantillonnage dans la première partie de l'étude était la méthode probabiliste aléatoire stratifié non proportionnelle, les deux strates présentent les deux EPS de Sfax où 433 infirmiers ont été sélectionnés par un tirage au sort (aléatoirement) et cela selon la figure suivante. 


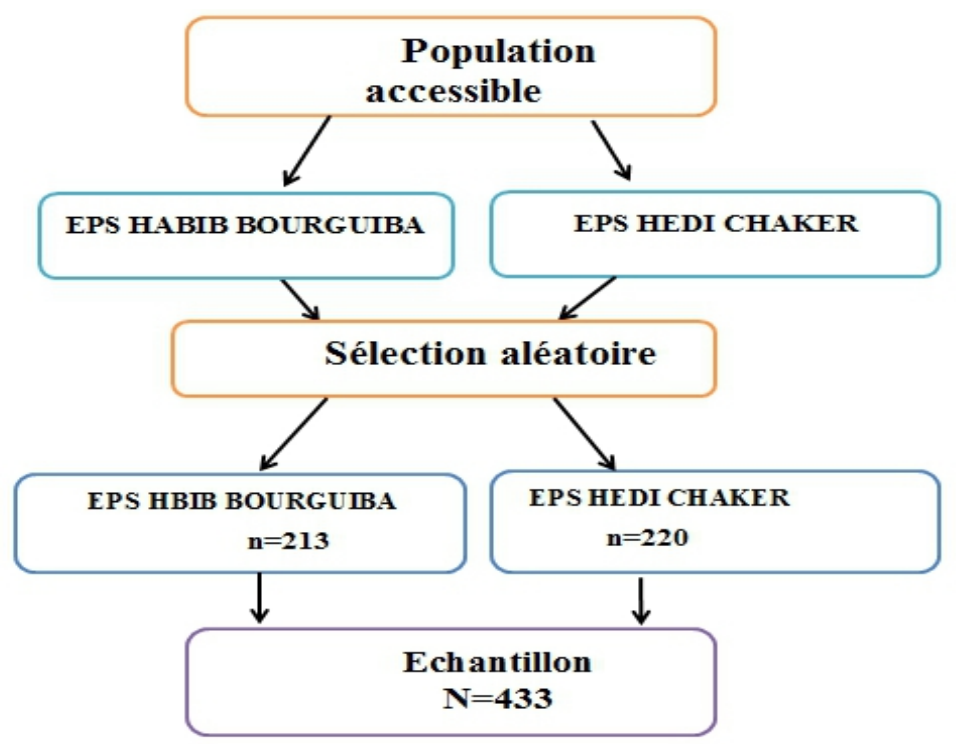

Figure 1 : Echantillonnage d'après la méthode de choix de la population et l'échantillon probabiliste stratifié non proportionnel.(Fortin M-F. 2010).

La deuxième partie de l'étude a suivi la méthode aléatoire afin de sélectionner aléatoirement 75 sujets parmi les $37.4 \%$ des participants qui équivaut à 160 infirmiers souffrant de burn-out (selon leurs score élevé au test du MBI et du questionnaire de Freudenberger) exerçant à plein temps, et ayant au moins trois ans d'expérience. Par contre, 60 seulement d'entre eux ont accepté de participer au programme proposé. Les participants se répartissent comme suit:

- 30 infirmiers au groupe expérimental : étant donné le nombre des participants, on a dû diviser le groupe expérimental en 3 sous-groupes dont chacun est composé de 10 infirmiers

- $\quad 30$ infirmiers au groupe témoin

- 09 infirmiers ont refusé de participer à l'étude

- 06 des participants se sont absentés aux séances programmées

Et pour mieux illustrer la méthode utilisée pour la sélection des participants à notre échantillon d'étude, le schéma suivant résume la démarche: 


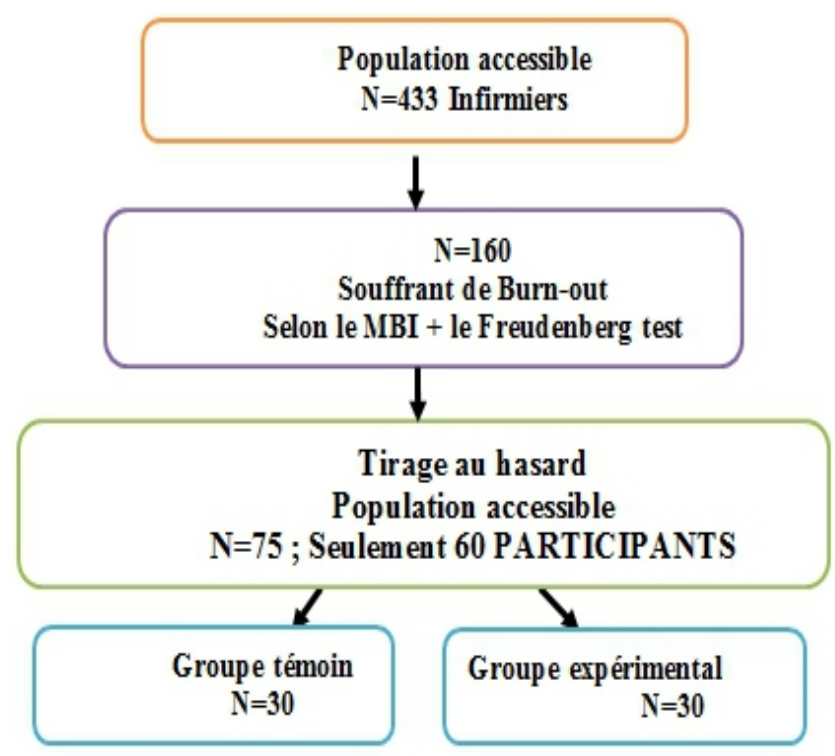

Figure2 : Echantillonnage aléatoire pour les deux groupes d'infirmiers souffrants de Burnout et participants à l'étude.

Le respect de l'homogénéité entre les deux groupes a été recherché autant que possible en veillant à ce que l'ensemble des participants :

-aient une expérience professionnelle de plus de 3 ans (important dans le diagnostic du burn-out) ;

-soient concernés par les permanences de nuit

-aient une ressemblance acceptable quant aux résultats des questionnaires d'auto évaluations lors du pré-test.

\section{Les étapes suivies au cours de cette recherche}

L'étude de terrain a été menée suivant plusieurs étapes :

la préparation d'un programme thérapeutique cognitivocomportemental visant à diminuer le burn-out en influençant les comportements, les cognitions et les émotions des infirmiers participants ;

- la sélection d'une batterie de tests évaluant les facteurs psychosociologiques (auto-efficacité, coping, motivation, satisfaction, le sens de la cohérence) ;

la sélection aléatoire simple des infirmiers du groupe expérimental souffrant de burn-out ;

- l'organisation des séances d'intervention thérapeutique selon un programme d'horaire qui respecte la disponibilité des infirmiers participant du groupe expérimental ; 
la mesure du degré de burn-out et les autres facteurs psychosociologiques lors d'un pré-test par l'application de la batterie de tests de la recherche afin de cerner ce syndrome ;

la mise en œuvre des séances du programme thérapeutique ;

la mesure lors d'un post-test en utilisant la même batterie de tests, pour vérifier l'efficacité du programme mis en œuvre par l'intervenante

la comparaison des résultats du pré-test et du post-test pour vérifier l'efficacité du programme thérapeutique cognitivo-comportemental.

\section{Déroulement de la collecte}

Pour l'étude descriptive, la collecte des données a été réalisée entre le $28 / 02 / 2013$, et le $05 / 05 / 2013$ et a concerné deux types de données :

- Les variables sociodémographiques et cliniques (sexe, âge, niveau d'instruction, ancienneté).

- $\quad$ La batterie des questionnaires burn-out et les indicateurs (l'estime de soi, la satisfaction, la motivation au travail, le coping, le locus of contrôle, le sens of cohérence et l'auto-efficacité) ont été remplis par 433 infirmiers durant leur travail (matin, après midi, nuit).

La durée des réponses au questionnaire a été estimée en moyenne à 15 minutes pour chaque infirmier.

La $2^{\text {ème }}$ partie a débuté en décembre 2013 et s'est prolongée à juin 2014. L'étude s'est déroulé auprès de 60 infirmiers des deux groupes : 30 pour le groupe expérimental et 30 pour le groupe témoin, l'application du programme était effectuée avec les infirmiers du groupe expérimental qui ont été répartis en 3 sous-groupes chaque sous-groupe comprenant 10 infirmiers.

Les séances ont suivi un timing précis : chaque semaine, les trois sousgroupes assistent à une séance

- $\quad$ Le 1er sous-groupe le mercredi après-midi

- $\quad$ Le 2ème sous- groupe le vendredi après-midi

- $\quad$ Le 3ème sous-groupe le samedi matin

\section{Instruments de mesure}

Le devis descriptif- expérimental de cette étude s'est basé sur deux techniques :

\section{Dans la première partie}

Qui a un but descriptif, une batterie de tests auto-administrés adaptée à ce sujet a été appliquée afin d'effectuer les mesures nécessaires en influençant les facteurs socio-psychologiques, et les déterminants indicateurs de ce syndrome ; estime de soi, auto-efficacité, satisfaction, motivation, coping, centre de contrôle, sens de la cohérence. Où les infirmiers ont été 
librement et anonymement sollicités pour remplir la batterie.Elle se compose de :

a) Une fiche de renseignement pour la collecte des données sociodémographiques, le genre, l’âge, l'état civil, le niveau socio-économique, le niveau d'instruction, la qualification professionnelle, l'ancienneté professionnelle et les problèmes au travail.

b) Les questionnaires et les échelles de mesures de burn-out et des facteurs d'influences standards et valides et fiables :

L'inventaire de burn-out de Maslach

Le questionnaire de burn-out de Freudenberger:

Le questionnaire d'estime de soi

Le Minnesota Satisfaction Questionnaire (MSQ) :

Le Multidimensional Work Motivation Scale (MWMS)

Le test de sens de la cohérence SOC :

Le test de sens de centre de contrôle:

L'échelle d'auto-efficacité :

Le questionnaire de coping:

\section{Dans la deuxième partie}

Le cadre d'expérimentation nécessite pour l'intervention l'application d'un programme thérapeutique visant à diminuer le burn-out par le traitement des facteurs de risques.

\section{a) Le programme thérapeutique cognitivo-comportemental :}

Au cours de ce programme thérapeutique, des entretiens de 24 séances (1 séance par semaine pour les 3 sous-groupes expérimentaux) ont été programmés et organisés tous les jeudis, vendredis et samedis pendant 12 semaines du 5 Janvier 2013 au 30 Mars 2013. Chaque séance a intéressé un groupe de 10 infirmiers. La durée de chaque séance est de $120 \mathrm{mn}$.

Le programme thérapeutique choisi se base sur la théorie cognitivocomportementale (TCC) qui s'intéresse à la partie visible de l'iceberg, la partie qui fait souffrir et, dans notre recherche: les comportements et les pensées problématiques liés au syndrome de burn-out.

Cette thérapie est centrée sur la cognition (pensées), les émotions et les comportements sans oublier les croyances parfois erronées et négatives que cultive l'individu sur lui-même. Le but de cette thérapie est de corriger ces pensées et croyances et de changer les comportements par de nouveaux comportements plus adaptés et plus adéquats. En général, ce sont des thérapies brèves qui s'appuient sur une relation active entre le thérapeute et son patient dans l'apprentissage de nouveaux comportements et la gestion des émotions.

Ces principes sont fondés sur l'apprentissage de nouveaux comportements, à partir de l'élaboration de pensées plus adéquates : il s'agit de réussir demain, ce dont on se croit incapable aujourd'hui et que, de ce fait, 
on a raté hier. Elle permet des changements positifs au niveau des souffrances psychiques ou physiques et elle favorise le développement personnel.

\section{b) Les phases du programme thérapeutique}

Le programme thérapeutique dans cette recherche s'inspire du travail de Cottraux (2011) et se réalise en 5 phases.

\section{$>\quad$ Une phase motivationnelle et éducative appelée aussi la psychoéducation:}

La psychoéducation vise d'abord à les informer de leur problématique, ce qui a souvent comme effet de les rassurer.

Elle vise également à présenter les avantages et les inconvénients du programme proposé par l'intervenant, à faire participer activement les membres du groupe à ce programme thérapeutique. Cette phase favorise la motivation, elle permet de mobiliser les participants et de les faire sortir du cercle vicieux de la démotivation (manque d'intérêts $\rightarrow$ inactivité $\rightarrow$ mauvaise image de soi $\rightarrow$ détérioration de l'humeur $\rightarrow$ encore moins d'intérêts et de motivation, etc.).

\section{$>\quad$ Une phase psychothérapeutique (techniques spécifiques comportementales et cognitives) :}

Elle consiste en l'utilisation de techniques de changement des comportements, des émotions et des pensées. Nous avons proposé des thèmes selon les principes qui régissent les interventions verbales cognitives favorisant la formulation des représentations, des questions, des doutes, des croyances dysfonctionnelles). Ensuite, un résumé des séances est fréquemment effectué et proposé à la discussion entre les participants, de manière à mettre à jour ce que le participant retient de la séance. En fin de la séance, il est demandé aux participants ce qui leur a déplu dans la séance et pourquoi, de manière à comprendre les lacunes, les erreurs et les problèmes.

\section{La phase de l'évaluation :}

Une évaluation des résultats obtenue au cours de la deuxième phase, elle vise à comprendre le dysfonctionnement. Elle peut également être utilisée pour diminuer certains comportements problématiques, comme l'isolement...etc.

Des tâches à domicile assurent la continuité du travail.

\section{$>\quad$ La phase de consolidation ou de résolution de problèmes:}

Elle permet au participant d'être le principal acteur dans la recherche de solutions à ses difficultés par la vérification de la compréhension et l'utilisation des techniques TCC apprises jusqu'alors ainsi que la régularité du style de vie et la gestion du stress individuel et interpersonnel.

\section{$>\quad$ Étape de la prévention des rechutes:}

Il est recommandé aux participants de revenir consulter s'ils sentent la reprise de leurs troubles au bout d'une quinzaine de jours. La chercheuse explique que les périodes chargées d'événements stressants peuvent agir sur 
leur vulnérabilité. Une reprise limitée de la thérapie effectuée à temps a de forte chance de résoudre ce problème.

c) Les composantes de la thérapie cognitivo-comportementale: $>$ L'analyse du dysfonctionnement cognitif émotionnel et comportemental:

La pratique repose en partie sur l'analyse du dysfonctionnement cognitif émotionnel et comportemental qui comprend une partie synchronique -actuelle -- et une partie diachronique - historique, et dans la présente étude, nous avons appliqué la 1 ère partie synchronique avec les sous - groupes expérimentaux qui a concerné l'état actuel de leur souffrance psychologique.

Il s'agit d'une anamnèse approfondie et structurée qui étudie les relations entre les comportements, les problèmes, les pensées, les émotions et l'environnement social professionnel de façon à adapter la démarche au participant et lui permettre, au travers d'une auto-observation, la mise à jour des déclencheurs (comme la situation problématique ou conflictuelle, les mécanismes mis en place face à cette situation (coping tel que l'automédication), l'attribution de cette situation ( interne ou externe), la cohérence des sujets, leur motivation, satisfaction et leur sentiment d'auto efficacité...), et bien sûr les monologues intérieurs y afférant, ainsi que les comportements "mécaniques".

\section{L'entraînement aux compétences par les techniques cognitivo- comportementales}

Il permet tout d'abord un travail cognitif, comportemental et émotionnel, encourage les participants à proposer des stratégies alternatives de résolution de problèmes, d'affirmation de soi, de faire face aux situations difficiles, de développer des pensées critiques et créatives...etc.

Vient ensuite l'élaboration d'objectifs pour chaque séance, qui consiste en un certain nombre de tâches précises à accomplir, car il s'agit d'une thérapie active où l'intervenante et les membres de groupe expérimental ont un rôle important, c'est-à-dire que l'intervenante motive les membres à participer tout au long des séances sans oublier qu'elle doit aussi être motivée, chaleureuse et positive à son tour.

D'où un autre principe essentiel aux TCC: une collaboration active entre les deux parties, qui permet d'évaluer ce que le participant peut accepter. Il est à signaler qu'il n'y a pas d'obligation donnée par l'intervenante.

L'intervenante, en collaboration avec les deux psychologues volontaires, utilisent des techniques précises basées sur des recherches scientifiques et empiriques qui visent à modifier favorablement les problèmes de la personne. Ces techniques sont les suivantes :

$\checkmark \quad$ Le modeling (et le modeling de participation)

$\checkmark \quad$ Le jeu de rôle (role playing) 
$\checkmark \quad$ Les tâches à domicile (HomeWork)

$\checkmark \quad$ Le renforcement positif (renforcer positivement la moindre réponse qui va dans le sens de l'amélioration et de l'épanouissement personnel au travail).

$\checkmark \quad$ L'affirmation de soi (l'entraînement aux habiletés sociales : entraîner les sujets à refuser les demandes exagérées qui émanent des patients et de leurs proches)

$\checkmark \quad$ L'exposition en imagination (situation de mort d'un patient, situation conflictuelle avec un médecin ou un collègue...)

$\checkmark \quad$ Des fiches à tenir en dehors des séances : pour décrire sentiment, comportement, et cognition en rapport avec le trouble (souffrance au travail), quand ? comment? où ? pourquoi ?

$\checkmark \quad$ Le disque rayé face à une demande de la part d'un patient ou d'un chef hiérarchique mécontent : cette technique consiste à répéter inlassablement la même phrase sur le même ton, répéter sans s'énerver, sans se justifier et sans donner d'arguments.

$\checkmark \quad$ Le brainstorming.

$\checkmark \quad$ La chaise haute, et la chaise vide.

\section{Plan d'analyse}

En lien avec les objectifs de la recherche, les analyses statistiques des données sont de type descriptif et corrélationnel. Les données issues du questionnaire ont été traitées à l'aide du logiciel statistique "Statistical Package for the Social Science » (SPSS, 20.0).

L'analyse comprend deux volets : le premier est descriptif (distribution de l'échantillon selon les pourcentages) qui concerne les données sociodémographiques et les résultats des questionnaires, le deuxième volet a trait à l'analyse statistique de comparaison de deux moyennes, les formules statistiques (la moyenne, l'écart type, variance) et le calcul du test de "t"Student de comparaison de moyennes des résultats du pré-test et post test qui a pour but de tester les hypothèses de notre recherche.

La partie suivante présente les résultats provenant des deux types d'analyse de données soit, les analyses descriptives et les analyses des tests paramétriques de comparaison de deux moyennes.

\section{Résultats}

Au niveau socio démographique, l'échantillon ( $\mathrm{N}=433)$ est marqué par un nombre hétérogène d'infirmiers et d'infirmières avec un sexe ratio de 0.89 . L'âge des participants varie entre $<30$ ans et 50 ans. Avec une moyenne d'âge $=36.75$. La plupart sont marié(e)s (61.9\%), la moitié (soit 52.4\%) appartient à la 2ème catégorie (soit une situation économique moyenne), (65\%) d'entre eux sont des bacheliers, (63\%) de l'échantillon ont le grade d'infirmier(e)s, 
(74.7\%) ont une ancienneté au travail qui varie entre (4ans et plus), pour les problèmes au travail le pourcentage le plus élevé était remarqué pour la charge de travail comme première source de stress ;ensuite le conflit et l'ambiguiité de rôle, l'absence de soutien, l'absence de contrôle ou d'autonomie dans l'exécution de la tâche. Après, on trouve en 5ème position les caractéristiques individuelles et enfin les participants classent les relations interpersonnelles en dernier lieu. Toutefois, l'enquête sur les obstacles rencontrés dans le cadre de l'exercice de leur travail a mis en évidence l'endroit du travail en premier et les conflits relationnels, suivis par les moyens de transport et une minorité a évoqué le manque de matériels et la charge de travail.

Les données utilisées ont été collectées auprès de 433 infirmiers choisis d'une manière probabiliste, aléatoire et stratifiée. Parmi cette population, les infirmiers qui souffrent de burn-out ont été sélectionnés pour participer au programme thérapeutique (en deux groupes; expérimental et témoin)

Les résultats obtenus à partir du questionnaire MBI de burn-out de Maslach indiquent que $37.4 \%$ des participants souffrent d'épuisement émotionnel élevé, $59.4 \%$ souffrent de dépersonnalisation élevée et $48.5 \%$ souffrent d'un sentiment de manque d'accomplissement dans leur travail. Ces résultats se rapprochent de ceux trouvés dans l'étude de Zarrad (2010) réalisée au service de psychiatrie en Tunisie, où les résultats du MBI ont montré que $39.5 \%$ avaient un score élevé d'épuisement émotionnel, toutefois, nos résultats sont nuancés par le fait que seulement $34.1 \%$ avaient une dépersonnalisation élevée et $17.5 \%$ avaient un accomplissement personnel bas.

L'étude de Maaroufi, Rzeigui, Ayari, Abid (2015) réalisée sur des soignants aux urgences, en Tunisie, a conclu que plus de la moitié de leur population $(56 \%)$ était touchée par le burn-out, $47 \%$ avaient un épuisement émotionnel élevé, $36,6 \%$ avaient un niveau élevé de dépersonnalisation et $33 \%$ avaient un niveau bas d'accomplissement professionnel. Le syndrome de burn-out était plus marqué chez les infirmiers par rapport aux médecins avec atteinte des trois dimensions.

Concernant l'estime de soi, près du tiers $(31 \%)$ de la population de l'étude avait un degré faible à très faible d'estime de soi.Selon André (2004), si celle-ci est faible, la culpabilité et l'autodénigrement engendrent autocritique et nervosité dans le travail qui donne plus de souffrances que de satisfactions.

Alors que $(72.5 \%)$ des participants avaient un niveau faible de sentiment d'auto-efficacité ce qui va à contre sens des résultats de l'étude de Nagels (2011) qui a trouvé un sentiment d'auto efficacité élevé chez les infirmiers de la population de son étude. 
Une motivation moyenne (soit 50.3\%) à faible (soit 44.8\%) avec $68.1 \%$ d'une faible motivation, $(44.8 \%)$ d'une faible motivation extrinsèque, $(47.3 \%)$ avaient une motivation intrinsèque faible,La motivation intrinsèque est considérée comme le plus haut niveau de motivation autodéterminée que peut atteindre un individu (Deci, 1975, cité dans Pelletier \&Vallerand, 1993). Elle est également la source d'énergie qui sert de départ à la nature active de l'organisme humain.

Les résultats ont confirmé que (70\%) étaient ni satisfaits/ ni insatisfaits, cela revient aux interactions entre les différents types d'adéquation entre la personne et son environnement professionnel dans une perspective temporelle lors de l'intégration dans l'emploi (Kristof-Brown, Bono \&Lauver, 1999).

Ainsi, (32\%)des participants avaient un niveau faible de sens de la cohérence, Ces résultats rejoignent ceux de l'étude d'Antonovsky (1974) qui affirment que $29 \%$ de la population étudiée (des femmes ayant vécu en camp de concentration) avaient un bon état de santé général, car pour Antonovsky le plus important ce n'est pas de comprendre ce qui rend l'individu vulnérable mais ce qui lui permet de se maintenir en bonne santé, bien qu'il soit soumis à des situations stressantes.

De plus, (52.9\%) des participants utilisaient des stratégies de coping centrées sur l'émotion et $(56.4 \%)$ cherchaient le soutien social alors que (75.3\%) utilisaient faiblement les stratégies de coping centrées sur la résolution de problèmes, Il est important de souligner que les réactions de la population étudiée sont surtout d'ordre émotionnel: comme consommer des substances addictives (alcool, tabac, drogues), s'engager dans diverses activités distrayantes (internet, lecture, télévision,...), exprimer ses émotions (colère, anxiété,...) et aussi chercher le soutien social.

Une majorité d'entre eux utilisait une attribution externe (44\%) due aux collègues $(42 \%)$ due au supérieur et aussi (42\%) due à la chance. Pour Rotter (1966), le contrôle interne est associé au coping positif et efficace, à une situation contrôlable, à de faibles perturbations psychologiques induites par les stresseurs (cité dans LaurencinePiquemal-Vieu, 2001).

Les hypothèses de la recherche stipulent qu'il existe une différence significative entre les données du pré-test et les données collectées au posttest chez les infirmiers du groupe expérimental. 
Tableau $\mathbf{n}^{\circ} \mathbf{1}$ : Résultats du Test-t Student pour échantillons appariés avec une distribution bilatérale des données statistiques de burn-out des infirmiers du groupe expérimental par le MBI et le FREUDENBERGER ( $\mathrm{N}=30)$

\begin{tabular}{|c|c|c|c|c|c|c|}
\hline \multirow{3}{*}{$\begin{array}{c}\text { Pré-test/post-test } \\
\text { Burn-out }\end{array}$} & \multirow{3}{*}{$\begin{array}{l}\text { Moyenne } \\
\text { des } \\
\text { différences }\end{array}$} & \multirow{2}{*}{\multicolumn{2}{|c|}{$\begin{array}{c}\text { Intervalle de } \\
\text { confiance } \\
99 \% \text { de la } \\
\text { différence }\end{array}$}} & \multirow[b]{3}{*}{$\mathrm{T}$} & \multirow[b]{3}{*}{ ddl } & \multirow{3}{*}{$\begin{array}{c}\text { Sig. } \\
\text { (bilatérale) }\end{array}$} \\
\hline & & & & & & \\
\hline & & Inf & Sup & & & \\
\hline MBI: épuisement émotionnel & 18 & 15,62 & 20,38 & 15,5 & 29 & 0 \\
\hline MBI: Dépersonnalisation & 12,17 & 10,74 & 13,59 & 17,47 & 29 & 0 \\
\hline $\begin{array}{l}\text { MBI: accomplissement } \\
\text { personnel }\end{array}$ & $-2,97$ & $-3,03$ & $-2,9$ & -89 & 29 & 0 \\
\hline TESTFreudenberger & 22,57 & 19,87 & 25,26 & 17,14 & 29 & 0 \\
\hline
\end{tabular}

D'après ce tableau, l'hypothèse de recherche est retenue car une diminution de burn-out chez les infirmiers qui ont participé à notre programme thérapeutique, selon le questionnaire de Maslach a été remarquée par les résultats de T Student.

De plus, la comparaison des résultats du pré-test /post-test des différentes variables du groupe expérimental a prouvé des différences significatives entre les variables traitées chez les infirmiers du groupe expérimental comme nous le montre le tableau suivant:

Tableau $n^{\circ} 2$ : Résultats du Test-t Student pour échantillons appariés avec une distribution bilatérale des données statistiques des variables de la recherche des infirmiers du groupe expérimental $(\mathrm{N}=30)$

\begin{tabular}{|c|c|c|c|c|c|c|}
\hline \multirow{3}{*}{$\begin{array}{l}\text { Pré-test/post-test du groupe } \\
\text { expérimental }\end{array}$} & \multirow{3}{*}{$\begin{array}{l}\text { Moyenne } \\
\text { des } \\
\text { différences }\end{array}$} & \multirow{2}{*}{\multicolumn{2}{|c|}{$\begin{array}{l}\text { Intervalle de } \\
\text { confiance } \\
99 \% \text { de la } \\
\text { différence }\end{array}$}} & \multirow[b]{3}{*}{$\mathrm{T}$} & \multirow[b]{3}{*}{ ddl } & \multirow{3}{*}{$\begin{array}{c}\text { Sig. } \\
\text { (bilatérale) }\end{array}$} \\
\hline & & & & & & \\
\hline & & Inf & Sup & & & \\
\hline Sens de la cohérence & 11,23 & 7,82 & 14,64 & 6,74 & 29 & 0 \\
\hline Estime de soi & $-8,73$ & $-10,52$ & $-6,94$ & $-9,98$ & 29 & 0 \\
\hline Motivation & $-67,6$ & $-72,32$ & $-62,88$ & $-29,32$ & 29 & 0 \\
\hline Satisfaction & $-52,67$ & $-55,17$ & $-50,17$ & $-43,07$ & 29 & 0 \\
\hline Coping centré sur le problème & $-20,83$ & $-22,75$ & $-18,92$ & $-22,26$ & 29 & 0 \\
\hline Coping centré sur l'émotion & 11,97 & 10,17 & 13,76 & 13,63 & 29 & 0 \\
\hline Coping centré sur le soutien social & $-2,97$ & $-4,21$ & $-1,72$ & $-4,87$ & 29 & 0 \\
\hline locus of contrôle: interne & $-3,47$ & $-4,24$ & $-2,69$ & $-9,13$ & 29 & 0 \\
\hline locus of contrôle: collègues & 4,87 & 4,68 & 5,06 & 52,53 & 29 & 0 \\
\hline locus of contrôle: supérieur & 3,83 & 3,02 & 4,65 & 9,62 & 29 & 0 \\
\hline locus of contrôle: chance & 4,73 & 4,24 & 5,22 & 19,77 & 29 & 0 \\
\hline Auto-efficacité & $-14,47$ & $-15,6$ & $-13,33$ & $-25,99$ & 29 & 0 \\
\hline
\end{tabular}

Les résultats de ce tableau expriment des différences significatives (au seuil de confiance de 0,01 ) au niveau des variables traitées au programme thérapeutique. Il s'en suit qu'il y a un changement au niveau du sens de la 
cohérence, de l'estime de soi, de leur sentiment d'auto-efficacité , de la motivation, de la satisfaction des stratégies de faire face a leurs problèmes, et de leurs centre de contrôle. Ce qui signifie l'efficacité du travail thérapeutique fourni au cours des séances.

Ainsi, les résultats de l'étude expérimentale ont répondu en général aux hypothèses de la recherche vu qu'ils ont prouvé des différences significatives entre les infirmiers des deux groupes (expérimental-témoin) au pré-test post-test, ce qui affirme l'efficacité du programme thérapeutique dans la diminution du burn-out et l'amélioration des indicateurs. En effet, la comparaison des résultats des différentes variables du post-test des deux groupes (expérimental/témoin) a été prouvé par les résultats statistiques comme nous le montre le tableau suivant :

Tableau n ${ }^{\circ}$ 3I : Résultats du Test-t Student pour échantillons indépendants de comparaison des moyennes au post-test des données statistiques du burn-out des deux groupes (expérimental et témoin) selon le MBI et le FREUDENBERGER

\begin{tabular}{|c|c|c|c|c|c|c|}
\hline \multirow{2}{*}{ Burn-out } & \multicolumn{5}{|c|}{ Test d'échantillons indépendants } \\
\cline { 2 - 7 } & $\begin{array}{c}\text { Test de Levene sur } \\
\text { l'égalité des } \\
\text { variances }\end{array}$ & \multicolumn{3}{|c|}{ Test-t pour égalité des moyennes } \\
\cline { 2 - 7 } & F & Sig. & T & ddl & $\begin{array}{c}\text { Sig. } \\
\text { (bilatérale) }\end{array}$ & $\begin{array}{c}\text { Différence } \\
\text { moyenne }\end{array}$ \\
\cline { 2 - 7 } & 3,11 & 0,083 & $-18,06$ & 58 & 0 & $-18,5$ \\
\hline L'épuisement émotionnel & 10,721 & 0,002 & $-16,89$ & 58 & 0 & $-11,4$ \\
\hline La dépersonnalisation & 0,076 & 0,783 & 7,04 & 58 & 0 & 8 \\
\hline $\begin{array}{c}\text { L'accomplissement } \\
\text { personnel }\end{array}$ & 1,279 & 0,263 & $-15,8$ & 58 & 0 & $-18,5$ \\
\hline Freudenberger & & & & & & \\
\hline
\end{tabular}

À la lumière des résultats de ce tableau, on remarque des différences significatives entre les infirmiers du groupe expérimental et ceux du groupe témoin après l'application du programme thérapeutique, car les moyennes du groupe expérimental sont significativement différentes, au seuil de confiance de 0,01 .

Tableau $\mathbf{n}^{\circ} 4$ : Résultats du Test-t Student pour échantillons indépendants de comparaison des moyennes au post-test des données statistiques des variables de la recherche des infirmiers des deux groupes (expérimental et témoin) au post test

\begin{tabular}{|c|c|c|c|c|c|c|}
\hline \multirow{2}{*}{$\begin{array}{c}\text { Résultats T Student des } \\
\text { deux groupes au post test }\end{array}$} & \multicolumn{6}{|c|}{$\begin{array}{c}\text { Test d'échantillons indépendants } \\
\text { l'égalité des } \\
\text { variances }\end{array}$} \\
\cline { 2 - 8 } & $\mathrm{F}$ & Sig. & $\mathrm{t}$ & $\mathrm{d}$ dl & $\begin{array}{c}\text { Sig. } \\
\text { (bilatérale) }\end{array}$ & $\begin{array}{c}\text { Test-t pour égalité des moyennes } \\
\text { moyenne }\end{array}$ \\
\cline { 2 - 8 } & 0,154 & 0,696 & $-7,12$ & 58 & 0 & $-11,7$ \\
\hline Sens de la cohérence & & & & & & \\
\hline
\end{tabular}




\begin{tabular}{|c|c|c|c|c|c|c|}
\hline Estime de soi & 0,426 & 0,516 & 11,9 & 58 & 0 & 8,6 \\
\hline Motivation & 0,07 & 0,792 & 39,25 & 58 & 0 & 68,6 \\
\hline Satisfaction & 11,796 & 0,002 & 36,94 & 58 & 0 & 52,9 \\
\hline Centré sur le problème & 0,417 & 0,521 & 20,1 & 58 & 0 & 20 \\
\hline Centré sur l'émotion & 3,183 & 0,08 & $-13,24$ & 58 & 0 & $-12,9$ \\
\hline Centré sur le soutien social & 0,024 & 0,878 & 3,57 & 58 & 0,001 & 2,5 \\
\hline Interne & 0,509 & 0,479 & 18,48 & 58 & 0 & 5,9 \\
\hline Collègues & 0,055 & 0,816 & $-21,86$ & 58 & 0 & $-4,8$ \\
\hline Supérieur & 3,178 & 0,08 & $-9,93$ & 58 & 0 & $-3,7$ \\
\hline Chance & 3,362 & 0,072 & $-16,12$ & 58 & 0 & $-4,7$ \\
\hline Auto-efficacité & 2,724 & 0,104 & 16,7 & 58 & 0 & 12,7 \\
\hline
\end{tabular}

À la lumière des résultats de ce tableau, on relève des différences significatives au niveau des variables étudiées chez les infirmiers des deux groupes après l'application du programme thérapeutique en faveur des infirmiers du groupe expérimental au seuil de confiance de 0,01 .

Cette étude apporte une perspective positive quant à la faisabilité des thérapies cognitivo-comportementales appliquées dans le domaine de la gestion du stress chronique et surtout de l'épuisement professionnel. Elles aident à la maitrise de soi et au développement des compétences et des habiletés personnelles puisque les infirmiers ont besoin de surmonter les situations conflictuelles et difficiles au travail et de développer des capacités à faire face et à résoudre le problème, surtout que l'infirmier d'aujourd'hui est accablé par divers obstacles au travail comme la surcharge (Lois Berry, IA, 2012).

\section{Conclusion}

Le burn-out reste un sujet de préoccupation majeure dans le travail des infirmiers. Cette étude de type descriptif expérimental utilise une approche quantitative visant en première partie à décrire ce syndrome ainsi que d'autres facteurs déterminants. En deuxième partie, évaluer l'efficacité d'un programme thérapeutique cognitivo-comportemental sur la diminution de la fréquence du syndrome du burn-out chez les infirmiers des centres hospitalouniversitaires de Sfax, par la comparaison des deux groupes expérimental et témoin concernant les données liées au burn-out à l'estime de soi, la satisfaction, la motivation, l'auto-efficacité, le sens de la cohérence, les stratégies de faire face (le coping), le centre de contrôle.

Notre recherche a réussi à répondre aux questions de recherche posées en ce qui concerne la description des concepts de l'étude ainsi qu'à l'efficacité du programme thérapeutique proposé. Les résultats ont prouvé que (37\%) des participants souffrent de burn-out. Notre étude a signalé des différences statistiquement significatives entre les infirmiers des deux groupes 
(expérimental et témoin) au pré-test et au post-test ce qui a confirmé les hypothèses de cette recherche.

Ainsi, on peut déduire que les infirmiers qui ont bénéficié de la prise en charge thérapeutique par TCC ont amélioré de leurs stratégies de coping, de leur centre de contrôle, de leur sens de la cohérence au travail, de leur estime et affirmation de soi, de leur satisfaction, de leur motivation et de leur sentiment d'efficacité.

$\mathrm{Au}$ terme de la présente étude, nous souhaitons vivement que les résultats viennent soutenir les infirmiers dans l'exercice de leur profession, en apportant un éclairage pertinent notamment du centre de contrôle, de la satisfaction au travail et de la motivation professionnelle, le coping, le sens de la cohérence, la performance au travail et leur estime de soi afin d'améliorer le climat de travail ainsi que la sécurité et la qualité de soins offertes aux patients.

\section{References:}

1. Cottraux. J (2001). Les thérapies cognitives. Comment agir sur nos pensées et nos émotions. Édition RETZ.

2. Cottraux. J (2011). Les psychothérapies comportementales et cognitives. ELSEVIER MASSON. 5 e Edition.

3. Cottraux, J .(2011). Choisir une psychothérapie efficace .Paris: Édition Odile Jacob.

4. Didier, T. (2004). Épuisement professionnel et Burn-out, concepts, modèles, interventions. Paris : Dunod.

5. Estryn-Béhar, M. (1997). Stress et souffrance des soignants à l'hôpital: Reconnaissance, analyse et prévention. De Boeck Supérieur.

6. Fortin M-F. (2010). Fondements et étapes du processus de recherche: méthodes quantitatives et qualitatives. 2e ed. Montréal:Chenelière Éducation.

7. Lois Berry, IA \& Curry, P. (2012). Charge de travail du personnel infirmier et soins aux patients. Publié par la Fédération canadienne des syndicats d'infirmières et infirmiers. Première édition.

8. Loriol, M. (2001). La fatigue, le stress et le travail émotionnel de l'infirmière. Revue Prévenir, 40 ,183-188.

9. Maslach, C \& Jackson S.E. (1986).Maslach burnout inventory manual. 2nd edition. Palo Alto: Consulting Psychologists Press, 34

10. Murtagh MJ, Thomson RG, May CR, Rapley T, Heaven BR, Graham RH, Kaner EF, Stobbart L, Eccles MP. (2007). Qualitative methods in a randomised controlled trial: the role of an integrated qualitative process evaluation in providing evidence to discontinue the intervention in one arm of a trial of a decision support tool. QualSaf Health Care;16(3):224-9. 
11. Pasquier-Garnier, G. (2012). Le Burnout ou épuisement professionnel, in psychologie de travail Information et prise en charge de la souffrance professionnelle. Université de Charles De Gaulle.

12. Phaneuf, M. (2002) .Communication, entretien, relation d'aide et validation, 12e chapitre :Prendre soin de soi pour prendre soin des autres, Montréal, Edition Chenelière Eductation /McGraw-Hill.

13. Poirier, M., \& Lafrenière A. (2003). la santé mentale des travailleurs : les perceptions des psychologues travaillantdans les PEA. Psychologie Québec, 14.

14. Rodary,C., Gauvain-Piquard,A.et al.(1993). Stress et épuisement professionnel.Objectifs Soins, 16,26-34.

15. Van Yperen, N.W., Buunk, B. P \&Schaufeli, W.B. (1992). Communal Orientation and the burnoutAmong Nurses. Journal of applied social psychology, 22, $173-189$. 